

${ }^{1}$ Retina Division,

Department of

Ophthalmology, Jules Stein Eye Institute, David Geffen School of Medicine at UCLA, Los Angeles, CA, USA

${ }^{2}$ Mechanical and Aerospace Engineering Department, Henry Samueli School of Engineering and Applied Science, UCLA, Los Angeles, CA, USA

${ }^{3}$ Center for Advanced Surgical and Interventional Technology (CASIT), Los Angeles, CA, USA

Correspondence: J-P Hubschman, Retina Division, Department of Ophthalmology, Jules Stein Eye Institute, UCLA, 100 Stein Plaza, Los Angeles, CA 90095-7000, USA.

Tel: +1 310206 5004;

Fax: +1 3107947905

E-mail: Hubschman@

jsei.ucla.edu

Received: 28 August 2012 Accepted in revised form: 24 April 2013

Published online: 31 May 2013

\section{Robot-assisted intraocular surgery: development of the IRISS and feasibility studies in an animal model}

E Rahimy' ${ }^{1}$ J Wilson², T-C Tsao ${ }^{2,3}$, S Schwartz ${ }^{1,3}$ and J-P Hubschman ${ }^{1,3}$

\begin{abstract}
Purpose The aim of this study is to develop a novel robotic surgical platform, the IRISS (Intraocular Robotic Interventional and Surgical System), capable of performing both anterior and posterior segment intraocular surgery, and assess its performance in terms of range of motion, speed of motion,

was successfully achieved in four eyes without any retinal tears/perforations noted. Conclusion Robotic-assisted intraocular surgery with the IRISS may be technically feasible in humans. Further studies are pending to improve this particular surgical platform. Eye (2013) 27, 972-978; doi:10.1038/eye.2013.105; published online 31 May 2013
\end{abstract} accuracy, and overall capacities. Patients and methods To test the feasibility of performing 'bimanual' intraocular surgical tasks using the IRISS, we defined four steps out of typical anterior (phacoemulsification) and posterior (pars plana vitrectomy (PPV)) segment surgery. Selected phacoemulsification steps included construction of a continuous curvilinear capsulorhexis and cortex removal in infusion-aspiration (I/A) mode. Vitrectomy steps consisted of performing a core PPV, followed by aspiration of the posterior hyaloid with the vitreous cutter to induce a posterior vitreous detachment (PVD) assisted with triamcinolone, and simulation of the microcannulation of a temporal retinal vein. For each evaluation, the duration and the successful completion of the task with or without complications or involuntary events was assessed.

Results Intraocular procedures were successfully performed on 16 porcine eyes. Four eyes underwent creation of a round, curvilinear anterior capsulorhexis without radialization. Four eyes had I/A of lens cortical material completed without posterior capsular tear. Four eyes completed 23-gauge PPV followed by successful PVD induction without any complications. Finally, simulation of microcannulation of a temporal retinal vein
Keywords: intraocular surgery; robot; phacoemulsification; vitrectomy

Introduction

Recent innovations in ophthalmology, namely the advent of small incision techniques and engineering of more efficient surgical devices, lend themselves to the mechanization of intraocular surgery. Consequently, one of the next major advancements in ophthalmology may be the integration of robotics.

Robotic systems have been utilized in the surgical environment for more than 15 years. During this period, robotic-assisted surgery has proliferated $^{1}$ in numerous disciplines, such as urologic ${ }^{2-5}$ gynecologic, ${ }^{6,7}$ and cardiovascular surgery. ${ }^{8-10}$ Several publications have reported equivalent efficiency and safety of roboticassisted procedures compared with conventional endoscopic techniques. ${ }^{11,12}$ The potential benefits of robotic-assisted surgery include increased precision and dexterity, elimination of tremor, task automation, and capacity for telesurgery.

The authors designed and built a surgical robotic platform dedicated to intraocular surgery named the 'IRISS' (Intraocular Robotic Interventional and Surgical System). The goal of this study was to assess the performance of the surgical platform in terms of range of motion, speed of motion, accuracy, and overall capacities. 


\section{Materials and methods}

\section{The robotic system}

The IRISS is a robotic surgical platform based on a master-slave manipulator design, and is dedicated for intraocular surgery. The master and slave communicate through a PXI system (National Instruments, Austin, TX, USA) running the LabVIEW Real-Time operating system with a sampling time of $1 \mathrm{~ms}$.

The master controller consists of two dedicated custom joysticks designed as master input devices. The surgeon holds both shafts and manipulates them as if they were standard intraocular surgical instruments. Motions of both shafts, measured by an optical encoder, are transmitted to the slave to mimic their movement. The scale between the master and the slave can be modified according to the difficulty of the task.

The slave manipulator includes two independent arms, each holding two automatically interchangeable surgical instruments, which are both mounted to a carriage riding on a circular track. The surgical instruments, mechanically constrained about a remote center of motion (RCM), or pivot point, have seven degrees of freedom (DoF) ( $\mathrm{z}$ direction, rotation, and the other two axes centered at the RCM $\theta \mathrm{x}$ and $\theta \mathrm{y}$, and cutting).

The two mechanical RCM are independent of one another and can be in close proximity (as less as $16 \mathrm{~mm}$ ), thus allowing for completion of 'bimanual' intraocular surgery. All types of commercially available microsurgical instruments can be adapted to fit on the surgical manipulator. In this study the surgical instruments attached to the surgical manipulator for cataract surgery evaluation were a viscoelastic syringe (Healon; Abbott Medical Optics, Abbott Park, IL, USA), capsulotomyirrigating needle (modified 30-gauge needle, B-D $30 \mathrm{G}$ 1/2, 305128; Becton, Dickinson \& Co., Franklin Lakes, NJ, USA), and an infusion-aspiration (I/A) probe (Alcon, Forth Worth, TX, USA). For vitreoretinal surgeries, a 23-gauge light pipe, a 23-gauge vitreous cutter, and a 23/36-gauge cannula (Alcon) were utilized. Except for the I/A probe, which was dedicated for research and animal study, the other surgical instruments were disposable.

\section{The visualization system}

Two different systems were tested for three-dimensional (3D) image acquisition during the surgical procedures. The first included a surgical microscope with a dual-port beam splitter (capture of the left-lens image and right-lens image) and two cameras (scout scA640-120fc; Basler, Exton, PA, USA). The second system tested was the TrueZoom 3D Surgical Camera with a $1920 \times 1080 \mathrm{p}$ resolution (TrueVision Systems, Santa Barbara, CA, USA).
The images were displayed and tested on two different visualization supports: (1) a 46-inch 1080 p 3D flat-panel LCD display with a high-definition (HD) resolution of $1920 \times 1080$ p $(1920 \times 540$ p per eye; TrueVision Systems $)$, and (2) a head-mounted display (model 920 AR; Vuzix, Rochester, NY, USA).

The four different combinations of 3D acquisition and displayed imaging systems were tested to assess the image resolution and the performances of the stereoscopic visualization; however, the specific combination of the TrueZoom 3D Surgical Camera plus the 3D 46-inch flat panel was used during the surgical procedures performed for this study.

\section{The range of motion, speed of motion, and stability of the RCM}

The mechanical range of motion of the surgical manipulators has been measured in the four DoF $(\theta x, \theta y$, $\theta \mathrm{z}$ and rotation) using CAD (SolidWorks, Waltham, MA, USA) model simulations. The range of motion of the main wrist and carriage mechanism is $\sim 120^{\circ}$. This is limited by component interference. The range of motion along the $\mathrm{z}$ direction of the surgical instrument (in and out) is $12.5 \mathrm{~cm}$. Finally, the range of motion of surgical instrument rotation is unlimited by the manipulator. Range of motion may be inhibited, however, by pneumatic tubes or electrical connections attached to the surgical instruments themselves.

The precision of the RCM for each robotic arm has been evaluated to view camera measurements of a calibration surgical instrument location as it is moved through the workspace. The RCM error due to instrument misalignment of the wrist joint and carriage joint was 1.66 and $0.59 \mathrm{~mm}$, respectively.

\section{Surgical performances in animal model}

To test the feasibility of performing 'bimanual' intraocular surgical tasks using the IRISS, we defined four separate steps out of typical anterior (phacoemulsification) and posterior (pars plana vitrectomy (PPV)) segment surgery. Selected phacoemulsification steps included construction of a continuous curvilinear capsulorhexis and cortex removal in I/A mode. Vitrectomy steps consisted of performing a core PPV, followed by aspiration of the posterior hyaloid with the vitreous cutter to induce a posterior vitreous detachment (PVD) assisted with triamcinolone, and simulation of the microcannulation of a temporal retinal vein. The microcannulation task consisted of applying pressure to the wall of a $200-\mu \mathrm{m}$ retinal temporal vein for $30 \mathrm{~s}$ with a 38-gauge needle (diameter of $100 \mu \mathrm{m}$ ). Both vitreoretinal tasks involved 'bimanual surgery,' meaning 
the simultaneous use of two instruments (vitreous cutter + light pipe and 38-gauge microcannula + light pipe). The visualization of the fundus was achieved using a disposable flat lens (Volk, Mentor, OH, USA). For each evaluation, the duration and the successful completion of the task with or without complications or involuntary events was assessed.

Procedures were performed on freshly collected porcine eyes affixed to a Styrofoam mannequin head. Given the absence of a motorized stage for both the robotic arms, the initial alignment of the surgical manipulator's RCM and ocular entry sites were done manually. Tasks were performed consecutively on a total of 16 porcine eyes: 4 eyes underwent anterior capsulorhexis, 4 eyes were subject to I/A of lens cortical material, 4 eyes underwent 3-port 23-gauge PPV followed by PVD induction, and 4 eyes were used to simulate microcannulation of a temporal retinal vein.

The porcine eyes were slaughterhouse materials, and the Institutional Animal Care and Use Committee of The University of UCLA approved the experimental protocol. All procedures were performed in the Center for Advanced Surgical and Interventional Technology at the UCLA Medical Center (Los Angeles, CA, USA). The telesurgical tasks were performed by a trained cataract and vitreoretinal surgeon (JPH).

\section{Results}

\section{Anterior capsulorhexis}

Four consecutive capsulorhexis attempts were created successfully using the IRISS (Supplementary Videos 1 and 2). Anterior capsular manipulations were smooth and accurate, with the formation of a round, curvilinear capsulorhexis each time. No radialization of the rhexis was encountered during any of the four attempts. The duration of each capsulorhexis was 205, 315, 259, and 319 s, respectively.

\section{Cortex removal in I/A mode}

Removal of lens cortical material via I/A mode was successfully performed using the IRISS in four consecutive instances (Supplementary Video 3). No anterior or posterior capsule tears were encountered during any of the attempts, which required 487, 331, 371, and $375 \mathrm{~s}$ to completion, respectively.

\section{3-Gauge PPV, including the detachment of the posterior hyaloid (PVD) assisted with triamcinolone}

Under high-magnification view, core vitrectomy was initially performed in four consecutive cases. A PVD was then successfully induced in all four instances without any complications. The duration of the procedure was $195,256,244$, and 327 s respectively.

\section{Simulation of the microcannulation of a temporal retinal vein}

Targeted retinal vessels had an inner diameter of $200 \mu \mathrm{m}$. The simulation of the microcannulation was achieved successfully in four consecutive cases (Supplementary Video 4). The 38-gauge microcannula was kept stationary and in contact with the created vascular wound in each attempt for at least $30 \mathrm{~s}$. No complications, specifically no retinal perforations, were observed after any of the attempts, which lasted $75,148,127$, and 88 s, respectively.

\section{Discussion}

Although the field of ophthalmic robotic surgery is still in its infancy, successful demonstrations of extraocular (corneal and scleral wounds), 13,14 anterior segment (foreign body removal and capsulorhexis), and posterior segment (25-gauge PPV) surgical tasks ${ }^{15}$ in animal models validate ongoing research efforts towards making it a clinical reality. While most surgical subspecialties have incorporated robotic platforms into routine use, ophthalmic surgery poses a number of unique engineering challenges that have thus far limited this technology's applicability. A microsurgical robotic system suitable for intraocular use must satisfy certain requirements with respect to the following: (1) ease of manoeuvrability within a confined workspace; (2) ability to execute motion of seven DoF; (3) RCM, or pivot point, located at the tissue entry sites; (4) stereoscopic visualization system; (5) efficient assembly/disassembly of sterile instrumentation from the unsterile platform; and (6) compatibility with the surgical environment (ie, assimilates to patient movement).

The da Vinci surgical system was previously evaluated because of its widespread acceptance as the premier robotic system on the market. ${ }^{15}$ Two design limitations, however, restrict its practical use in ophthalmic surgery. First, having a high RCM, both located above the wrist and at a long distance from the tip of the instrument, renders intraocular maneuvers less controllable and induces undue tension on the external eye surface next to the entry site. Thus, the surgeon is required to impose a second RCM at the ocular penetration site by moving the robotic arms appropriately. These motions are not as intuitive as the wrist movements, do not mirror the exact movements of the surgeon's arms, and, more importantly, dramatically limit the range of motion. Consequently, creation of a perfectly round, curvilinear capsulorhexis optimal for cataract surgery has proven to 
be quite difficult. ${ }^{15}$ Second, intraoperative visualization as ophthalmologists are accustomed to is challenging with the da Vinci surgical system as its video capture system is designed for endoscopic use and does not yield the detail of a sophisticated optical microscope.

These shortcomings of the da Vinci surgical system have spurred efforts by several teams towards customizing platforms solely dedicated for intraocular surgery. By mounting a microrobot, the Hexapod Surgical System, to the da Vinci macrorobot, an RCM located at the site of ocular penetration was achieved. ${ }^{16}$ Another adaptation, the Microhand (consisting of four fingers), was designed to mimic a human hand while being pneumatically controlled, allowing titration of grasping force. ${ }^{17}$ The steady-hand manipulator ${ }^{18,19}$ and Micron $^{20}$ were two independently developed instruments capable of tremor filtration for microsurgical procedures. Japanese collaborators recently created a prototype robotic system designed to aid in vitreoretinal surgery. Robotic assistance increased surgeon accuracy 5- to 10-fold, and facilitated successful surgical induction of a PVD, retinal vessel sheathotomy using 25-gauge microscissors, and microcannulation of retinal vessels in porcine eyes. ${ }^{21}$ A separate group of investigators have designed a novel dual-arm robot capable of highprecision maneuvers, such as vascular cannulation and stent deployment in animal models. ${ }^{22,23}$

Despite these numerous achievements, predecessors of the IRISS have primarily focused on the execution of single tasks, or assisting in technically difficult portions of procedures. However, no system has been capable of performing a complete ocular surgical procedure, including both anterior and posterior intraocular surgeries. Interest is growing in designing such devices, with the goal of adding speed and efficiency to the surgical experience without sacrificing precision. The IRISS, a joint effort between the Jules Stein Eye Institute and the UCLA Department of Mechanical and Aerospace Engineering, represents the first such dedicated microsurgical platform capable of performing complete ophthalmic procedures, including both anterior and posterior segment surgery.

The IRISS design consists of two components: a master controller and a dual-arm surgical instrument slave manipulator, set on different tables in the same operating suite (Figure 1). Each arm of the mechanical robot apparatus is mounted on a separate stage with its own RCM (Figure 2), capable of seven DoF necessary for intraocular manipulation: three translational DoF to align the tip of the instrument to the incision site; two rotational and one translational DoF during the intraocular operation; and one more DoF for the forceps or other instruments (ie, scissors, vitreous cutter, and phaco probe). In addition, each arm is equipped with interchangeable commercially available instruments, enabling efficient mid-surgery switching for completion of bimanual tasks. As most intraocular procedures require the use of two instruments simultaneously inside the eye, each of the two RCM must be independently enforced at different locations on the ocular surface. Because of the unique space confines in and around the globe, these RCM must also be in close proximity to each other, while being able to move in order to align the manipulator with the incision point during surgery, if necessary. The relative orientation of the two surgical instruments can vary from almost parallel during posterior segment surgery to antiparallel during cataract surgery. The IRISS manipulator is unique as it has a range of motion large enough to accommodate both anterior and posterior surgical procedures, while positioning the RCM associated with each arm less than $16 \mathrm{~mm}$ apart from each other. Of note, the IRISS platform's RCM is mechanically enforced, providing increased accuracy and safety of the task motions compared with a virtual RCM achieved by using a software to coordinate robot joints.

The control console where the surgeon manipulates the robotic arms remotely consists of two custom-designed



Figure 1 Experimental setup of the IRISS.

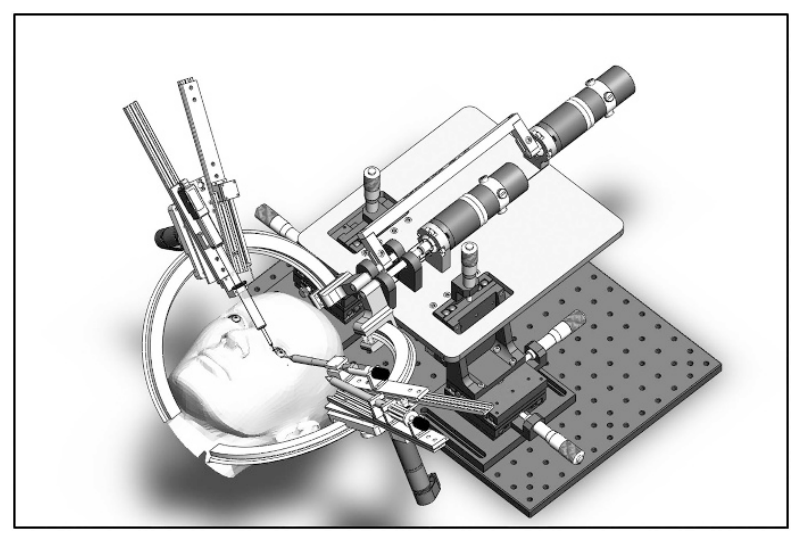

Figure 2 Computer-aided design of the IRISS slave manipulator. Each arm of the robot apparatus is mounted on a separate stage with its own remote center of motion. 
joystick controls. The surgeon holds both shafts and manipulates each as if they were standard intraocular surgical instruments. Tremor reduction is achieved by processing and filtering frequency of motion. Small motion can be amplified from the joystick command to the instrument so that surgical precision is increased. The system is not subject to fatigue. Optimal model basecontrol algorithms implemented help mitigate vibration at the surgical instrument due to manipulator flexibility in the presence of motor friction. The need for a superior image acquisition and visualization system, capable of delivering a $3 \mathrm{D}$ view to the surgeon seated away from the microscope, was satisfied with the TrueZoom 3D Surgical Camera (TrueVision Systems) mounted on the microscope. Enhanced HD 3D visualization was possible by either a head-mounted or 3D flat-panel display.

Motivated by the goal to perform entire intraocular procedures, four surgical tasks, representative of both anterior and posterior segment surgery, were selected to demonstrate the different features needed for an intraocular robotic platform: range of motion, dexterity, and accuracy. We previously determined the range of motion required to carry out common intraocular surgical tasks by attaching electromagnetic sensors (MicroBird; Ascension Technology, Burlington, VT, USA) to instruments used in cataract surgery (ie, phacoemulsification handpiece and cataract chopper) and vitrectomy surgery (ie, vitreous cutter and intraocular light pipe), which then quantified microscopic translational and angulational movements. ${ }^{24}$ That study's results showed that robotic ocular surgery devices holding instruments should be designed to allow a minimum translation of $3.65,3.14$, and $2.06 \mathrm{~cm}$ in the $\mathrm{x}, \mathrm{y}$ and z-planes, respectively, whereas a minimum angulation of 116 and $106^{\circ}$ were needed intraocularly in the $x$ - and $y$-planes. ${ }^{24}$

Although three of the employed tasks (anterior lens capsulorhexis, I/A of the lens material, and 23-gauge core PPV with PVD induction) were clinically relevant proceedings, the fourth (retinal vein microcannulation) was evaluated because it is a technically difficult and delicate maneuver to assess our prototype with. Previous reports state that the maximum hand tremor of vitreoretinal surgeons during intraocular procedures exceeds $100 \mu \mathrm{m} \cdot{ }^{21,25-27}$ However, microcannulation into retinal vessels with a diameter of $\sim 200 \mu \mathrm{m}$ would require an accuracy of nearly $10 \mu \mathrm{m}$. Successful completion of such a task may be indicative of the robot's potential to perform other high-dexterity functions, such as an epiretinal membrane peel.

Despite the longer duration, each attempt per task was accurately and reproducibly completed without complication. Furthermore, in contrast with previous attempts on the da Vinci surgical system, ${ }^{15}$ the IRISS is the first robotic system to not only successfully create a round, curvilinear capsulorhexis essential for cataract surgery, but also the first to carry out an entire cataract extraction from start to finish. As this study demonstrated, the IRISS displayed the needed range of motion, dexterity, and accuracy for delicate intraocular surgery.

While using the IRISS to perform the surgical procedures previously discussed, the most difficult task encountered was manually aligning the surgical manipulator RCM to the incision made in the porcine eye. This trial and error process was time consuming and needed to be performed to compensate for any relative movement between the eye and the surgical manipulator. This would preclude the clinical use of the IRISS in its current state, as the patient is not completely anesthetized and minimally restrained by tape across the forehead, rendering even the slightest head movements potentially detrimental. Therefore, incorporation of a tracking system and motorization of the two stages to compensate for the eye movement will be the next logical steps in refining the IRISS for potential use on living subjects.

Recently, laser refractive surgery has dramatically evolved to become a nearly completely automated process, requiring minimal intraoperative manipulation by the surgeon. Similarly, we anticipate future adaptations to the IRISS platform may contribute to the inevitable shift towards automation of other anterior segment microsurgical procedures, namely cataract surgery, by enhancing surgical capacities and safety, while reducing operating time. This may plausibly be achieved by integrating the IRISS with a femtosecond laser device, which has been optimized for wound construction, capsulorhexis creation, and nuclear fragmentation during cataract surgery. In addition, assimilation of the IRISS platform with intraoperative visual recognition technology, optical coherence tomography, ${ }^{28-30}$ and/or laser technology could help facilitate creation of a 'no-fly zone,' whereby certain vital intraocular structures (ie, posterior capsule) can be delineated and restricted from instrumentation. For less standardized procedures, such as vitreoretinal surgery, robotic augmentation may potentially improve surgical outcomes by increasing surgeon dexterity and accuracy, decrease complication rates by reducing tremor, and open the door for more delicate, fine-detail surgical manipulations by amplifying scale of motion.

Perhaps the most intriguing aspect of robotic ocular microsurgery is the capability to perform procedures from a remote location (telesurgery). Transatlantic robotic-assisted surgery has already been experimentally demonstrated in other specialties. ${ }^{31,32}$ Given the everincreasing ocular surgical demand globally, telesurgery affords a unique opportunity to bring the surgeon to the 
patient rather than having the patient come to the surgeon. As a result, delivering complex and delicate ophthalmologic surgical services in locations with limited access to specialty care may one day be a feasible consideration.

Several obstacles remain before robotic surgery can become a clinical reality in ophthalmology. Justification of high costs, potential steep learning curves for the novice surgeon, and patient trust all present unique challenges. Regardless, ophthalmic surgery has the potential to be revolutionized by robotic platforms, and research efforts should continue forward, provided future robotic systems will surpass current limitations of human-delivered surgical care. Here, we present a preliminary evaluation of the IRISS, which represents, to our knowledge, the first novel intraocular robotic prototype capable of performing complete anterior and posterior segment surgeries. Although the results are encouraging, additional refinements are underway, as we aim to translate this system from the bench-top to the operating room.

\section{Summary}

What was known before

- Ophthalmic surgery poses a number of unique engineering challenges that have limited the applicability of robotic-assisted technology to date.

- Previous ocular surgical robotic platforms/ instrumentation primarily focused on the execution of single tasks, or assisting in technically difficult portions of procedures. However, no system has been capable of performing a complete ocular surgical procedure, including both anterior and posterior intraocular surgeries.

\section{What this study adds}

- The IRISS represents the first dedicated microsurgical platform capable of performing complete ophthalmic procedures, including both anterior and posterior segment surgery

- It is the first robotic system to not only successfully create a round, curvilinear capsulorhexis essential for cataract surgery, but also the first to carry out an entire cataract extraction from start to finish.

- Despite the longer time duration for each task completed, the IRISS displayed the needed range of motion, dexterity, and accuracy for delicate intraocular surgery.

- Robotic-assisted intraocular surgery with the IRISS may be technically feasible in humans. Additional refinements are underway to improve this surgical platform and prepare it for future clinical use.

\section{Conflict of interest}

The authors declare no conflict of interest.

\section{References}

1 Buckingham RA, Buckingham RO. Robots in operating theatres. BMI 1995; 311: 1479-1482.

2 Ruurda JP, Broeders IA, Simmermacher RP, Borel Rinkes IH, Van Vroonhoven TJ. Feasibility of robot-assisted laparoscopic surgery: an evaluation of 35 robot-assisted laparoscopic cholecystectomies. Surg Laparosc Endosc Percutan Tech 2002; 12: 41-45.

3 Kumar R, Hemal AK. Emerging role of robotics in urology. J Minim Access Surg 2005; 1: 202-210.

4 Dasgupta P, Challacombe B, Murphy D, Khan MS. Coming full circle in robotic urology. BJU Int 2006; 98: 4-5.

5 Kaul S, Laungani R, Sarle R, Stricker H, Peabody J, Littleton $\mathrm{R}$ et al. da Vinci-assisted robotic partial nephrectomy: technique and results at a mean of 15 months of follow-up. Eur Urol 2007; 51: 186-191; discussion 191-182.

6 Diaz-Arrastia C, Jurnalov C, Gomez G, Townsend Jr C. Laparoscopic hysterectomy using a computer-enhanced surgical robot. Surg Endosc 2002; 16: 1271-1273.

7 Beste TM, Nelson KH, Daucher JA. Total laparoscopic hysterectomy utilizing a robotic surgical system. JSLS 2005; 9: 13-15.

8 Katz MR, Van Praet F, de Canniere D, Murphy D, Siwek L, Seshadri-Kreaden U et al. Integrated coronary revascularization: percutaneous coronary intervention plus robotic totally endoscopic coronary artery bypass. Circulation 2006; 114: I473-I476.

9 McClure RS, Kiaii B, Novick RJ, Rayman R, Swinamer S, Kodera $\mathrm{K}$ et al. Computer-enhanced telemanipulation in mitral valve repair: preliminary experience in Canada with the da Vinci robotic system. Can J Surg 2006; 49: 193-196.

10 Kypson AP, Chitwood WR. Robotic cardiovascular surgery. Expert Rev Med Devices 2006; 3: 335-343.

11 Breitenstein S, Nocito A, Puhan M, Held U, Weber M, Clavien PA. Robotic-assisted versus laparoscopic cholecystectomy: outcome and cost analyses of a casematched control study. Ann Surg 2008; 247: 987-993.

12 Zorn KC, Gofrit ON, Orvieto MA, Mikhail AA, Galocy RM, Shalhav AL et al. Da Vinci robot error and failure rates: single institution experience on a single three-arm robot unit of more than 700 consecutive robot-assisted laparoscopic radical prostatectomies. J Endourol 2007; 21: 1341-1344.

13 Bourges JL, Hubschman JP, Burt B, Culjat M, Schwartz SD. Robotic microsurgery: corneal transplantation. Br J Ophthalmol. 2009; 93: 1672-1675.

14 Tsirbas A, Mango C, Dutson E. Robotic ocular surgery. Br J Ophthalmol. 2007; 91: 18-21.

15 Bourla DH, Hubschman JP, Culjat M, Tsirbas A, Gupta A, Schwartz SD. Feasibility study of intraocular robotic surgery with the da Vinci surgical system. Retina 2008; 28: 154-158.

16 Bourges JL, Hubschman JP, Wilson J, Prince S, Tsao TC, Schwartz S. Assessment of a hexapod surgical system for robotic micro-macro manipulations in ocular surgery. Ophthalmic Res 2011; 46: 25-30.

17 Hubschman JP, Bourges JL, Choi W, Mozayan A, Tsirbas A, Kim CJ et al. 'The Microhand': a new concept of micro-forceps for ocular robotic surgery. Eye (Lond) 2010; 24: 364-367.

18 Mitchell B, Koo J, Iordachita I, Kazanzides P, Kapoor A, Handa $\mathrm{J}$ et al. Development and application of a new steady-hand manipulator for retinal surgery. IEEE ICRA 2007; 623-629. 
19 Uneri A, Balicki MA, Handa J, Gehlbach P, Taylor RH, Iordachita I. New steady-hand eye robot with micro-force sensing for vitreoretinal surgery. Proc IEEE RAS EMBS Int Conf Biomed Robot Biomechatron 2010; 2010: 814-819.

20 MacLachlan RA, Becker BC, Tabarés JC, Podnar GW, Lobes LA, Riviere CN. Micron: An actively stabilized handheld tool for microsurgery. IEEE Transactions on Robotics 2012; 28: 195-212.

21 Ueta T, Yamaguchi Y, Shirakawa Y, Nakano T, Ideta R, Noda $Y$ et al. Robot-assisted vitreoretinal surgery: development of a prototype and feasibility studies in an animal model. Ophthalmology 2009; 116: 1538-1543.

22 Fine HF, Simaan N, Wei W, Siman N. A novel dual-arm dexterous ophthalmic microsurgical robot: applications for retinal vascular cannulation and stent deployment. Retina Congress 2009.

23 Fine HF, Wei W, Goldman R, Simaan N. Robot-assisted ophthalmic surgery. Can J Ophthalmol 2010; 45: 581-584.

24 Hubschman JP, Son J, Allen B, Schwartz SD, Bourges JL. Evaluation of the motion of surgical instruments during intraocular surgery. Eye (Lond) 2011; 25: 947-953.

25 Riviere CN, Rader RS, Khosla PK. Characteristics of hand motion of eye surgeons. Conf Proc IEEE Eng Med Biol Soc 1997; 4: 1690-1693.
26 Riviere CN, Jensen PS. A study of instrument motion in retinal microsurgery. Conf Proc IEEE Eng Med Biol Soc 2000; 1: 59-60.

27 Singh SP, Riviere CN. Physiological tremor amplitude during retinal microsurgery. Proc IEEE Northeast Bioeng Conf 2002; 171-172

28 Liu X, Balicki M, Taylor RH, Kang JU. Automatic online spectral calibration of Fourier-domain OCT for robotic surgery. Proc SPIE 2011; 7890: 875210.

29 Liu X, Balicki M, Taylor RH, Kang JU. Towards automatic calibration of Fourier-domain OCT for robot-assisted vitreoretinal surgery. Opt Express 2010; 18: 24331-24343.

30 Balicki M, Han JH, Iordachita I, Gehlbach P, Handa J, Taylor R et al. Single fiber optical coherence tomography microsurgical instruments for computer and robot-assisted retinal surgery. Med Image Comput Comput Assist Interv 2009; 12: $108-115$.

31 Marescaux J, Leroy J, Gagner M, Rubino F, Mutter D, Vix M et al. Transatlantic robot-assisted telesurgery. Nature 2001; 413: 379-380.

32 Marescaux J, Leroy J, Rubino F, Smith M, Vix M, Simone M et al. Transcontinental robot-assisted remote telesurgery: feasibility and potential applications. Ann Surg 2002; 235: 487-492.

Supplementary Information accompanies this paper on Eye website (http://www.nature.com/eye) 